\title{
Are the number of teeth any help in assessing development?
}

The concept of dental maturity or 'dental age' has existed in some shape or other since the 1920 s. Mostly it has been based on the number of teeth present in the mouth at each chronological age. Although the use of dental emergence is attractively simple, there are inherent limitations: (i) while the method may be applicable for population groups there is a high possibility of inaccuracies for any individual child; (ii) there are quite long periods when there is no variation in the number of teeth and thus very little information about maturation; and (iii) local pathological states may drastically influence the picture.

When there is more detailed information about the age of emergence of a number of teeth this type of method may be refined as, for example, by Filipsson. ${ }^{1}$ In his method an average profile of the age of emergence of the first 12 permanent teeth was developed for the population. Then for a given child, where the age of emergence of all or some of the first 12 teeth are known, the eruption pattern of the child's teeth is fitted to the average profile and what is in effect a prediction of the age of eruption of the 12 th tooth made. Thus a child with a relatively delayed dental maturity will give a somewhat older age for this prediction and the relatively advanced child an earlier one.

Since the early 1950 s a different concept of dental maturity has been gaining ground. In this, the concept is extended to take account of the calcification of a tooth, greatly extending the information content. While the emergence of a tooth is a fleeting event and it may be impossible to determine its exact time, the calcification is a continuous process that may be assessed from permanent records such as radiographs.

This process of assessing dental maturity is very much like the assessment of skeletal maturity from radiographs of the hand and wrist. In a way similar to the bones of the hand, teeth undergo a sequence of maturational stages. The first is the formulation of the crypt and the final stage is the fully mature tooth. During this process continuous changes may be seen in the size and shape of the tooth. Each tooth follows the same sequence, and in order to study the entire process arbitrary stages must be selected to trace the entire developmental process from beginning to end. Unlike the skeleton, the dental system has two overlapping developmental periods for two sets of teeth. That for the deciduous teeth extends from the third month of intrauterine life to the third year of postnatal life. For the permanent teeth it is from the age of 6 months (postnatal) to about 15 years, if the third molars are excluded.

A number of radiographic methods have been described, some depending upon a single tooth and others on many. A reasonable compromise seems to be the method described by Demirjian, Goldstein, and Tanner ${ }^{2}$ which depends on recognising up to 8 developmental stages in 7 teeth; first and second molars, first and second bicuspids, canine, lateral incisor, and central incisor. These ratings are made on a panoramic radiograph of the maxilla and mandible that gives a complete view of the relevant dentition. The teeth are evaluated, a score is attributed to each, and then a total dental maturity score between 0 and 100 is determined. This may be converted to a dental 'age' in a way entirely analogous to a skeletal 'age' in the method of bone ageing described by Tanner et $a .^{3}$ The method may be simplified to allow use of only four teeth.

To answer the question raised in the title, we now need to evaluate whether assessment of dental age by one of the emergence methods or by a radiographic technique tells us anything about development. Here is a trap for the unwary, in that if one looks at correlations between dental maturity (by either method) and, for example, bone age across a large span of chronological age, quite high correlations will be found. It would then be easy to say that dental age and skeletal age are highly correlated and that dental maturation may be used as a measure of skeletal or other development. If children in such a study are divided into relatively narrow bands of chronological age, then the correlation between dental and skeletal maturity almost evaporates. The point is that if there are two processes of maturation continuing in parallel and the correlation between the two methods is assessed over a relatively long time span they will inevitably seem to be closely associated. They may, however, be quite unrelated in their more detailed relation, simply reflecting the passage of time: knowing something about dental maturation need tell you very little about general maturation.

This prediction is indeed borne out. When age 
specific correlations are studied between different maturity indicators and dental age the correlations are in general very poor. The independence between bone and tooth maturation is, in fact, not that surprising. The two have quite different embryological origins. Teeth are at least partially of epithelial origin while the bones are derived entirely from mesoderm. In many ways it is much more sensible to consider these two maturity indicators together with other common ones such as puberty ratings and, for that matter, developmental stages of intellectual function as just reflecting an overall tempo of maturation of the child. Each maturity indicator tells us a little about this overall process and, to a varying degree, overlaps with other methods of assessment. However, no one method tells us everything about another, and for this reason the answer to the original question is essentially 'not very much'.

References

1 Filipsson R. A new method for assessment of dental maturity using the individual curve of number of erupted permanent teeth. Ann Hum Biol 1975;2:13-24.

2 Demirjian A, Goldstein H, Tanner JM. A new system of dental age assessment. Hum Biol 1973 ;45:211-27.

3 Tanner JM, Whitehouse RH, Marshall WA, Healy MJR, Goldstein H. Assessment of skeletal maturity and prediction of adult height: TW2 method. London: Academic Press, 1975.

M A PREECE

Institute of Child Health, 30 Guilford Street, London WC1N 1EH 